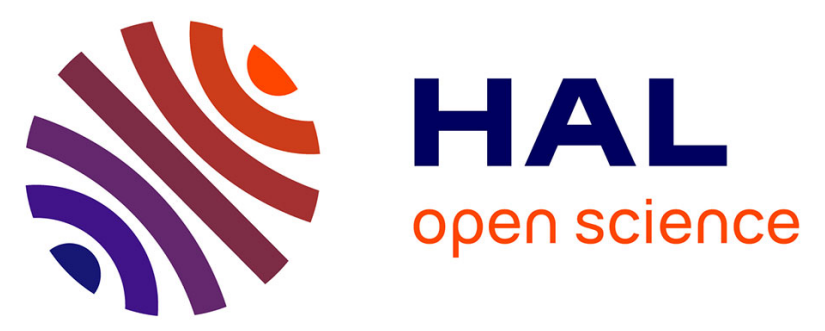

\title{
Photolariats: synthesis, metal ion complexation and photochromism
}

Aurélien Ducrot, Peter Verwilst, Luca Scarpantonio, Sébastien Goudet, Brice Kauffmann, Sergey A. Denisov, Gediminas Jonusauskas, Nathan D.

Mcclenaghan

\section{To cite this version:}

Aurélien Ducrot, Peter Verwilst, Luca Scarpantonio, Sébastien Goudet, Brice Kauffmann, et al.. Photolariats: synthesis, metal ion complexation and photochromism. Supramolecular Chemistry, 2012, 24 (4), pp.462-472. 10.1080/10610278.2012.678851 . hal-00732071

\section{HAL Id: hal-00732071 \\ https://hal.science/hal-00732071}

Submitted on 30 Aug 2018

HAL is a multi-disciplinary open access archive for the deposit and dissemination of scientific research documents, whether they are published or not. The documents may come from teaching and research institutions in France or abroad, or from public or private research centers.
L'archive ouverte pluridisciplinaire HAL, est destinée au dépôt et à la diffusion de documents scientifiques de niveau recherche, publiés ou non, émanant des établissements d'enseignement et de recherche français ou étrangers, des laboratoires publics ou privés.

\section{(1)(1) $\$(0)$}

Distributed under a Creative Commons Attribution - NonCommercial - ShareAlikel 4.0 


\title{
Photolariats: synthesis, metal ion complexation and photochromism
}

\author{
Aurélien Ducrot ${ }^{\mathrm{a}}$, Peter Verwilst ${ }^{\mathrm{a}}$, Luca Scarpantonio ${ }^{\mathrm{a}}$, Sébastien Goudet ${ }^{\mathrm{a}}$, Brice Kauffmann ${ }^{\mathrm{b}}$, Sergey Denisov ${ }^{\mathrm{a}, \mathrm{c}}$, \\ Gediminas Jonusauskas ${ }^{c}$ and Nathan D. McClenaghan ${ }^{\mathrm{a} *}$ \\ ${ }^{a}$ Institut des Sciences Moléculaires, University of Bordeaux/CNRS, 351 crs de la Libération, Talence 33405, France; ${ }^{b}$ Institut Européen \\ de Chimie et Biologie, University of Bordeaux, 2 Rue Robert Escarpit, Pessac 33607, France; ${ }^{c}$ Laboratoire Ondes et Matière \\ d'Aquitaine, University of Bordeaux/CNRS, 351 crs de la Libération, Talence 33405, France
}

\begin{abstract}
Photolariat development, as an extension to the family of synthetic photochromic crown ether receptors, or photocrowns, is reported. Incorporated additional chelating groups, namely two anisoles or thioanisoles, contribute in completing the metal ion coordination sphere with different affinities and selectivities for a range of ions. Single crystal X ray diffraction analysis suggests that the thermally stable trans form of the receptor is unsuitable for ion binding, consistent with spectrophotometric and NMR titration results, which is largely improved in the cis form as the basis for the photocontrolled ion coordination/ejection. In terms of the azobenzene containing receptor photochemistry, a photostationary state highly enriched in the cis form (94:6, cis /trans ) is reached, with slow thermal return $\left(1.11 .4 \times 10^{5} \mathrm{~s}^{1}\right)$ in the dark, which can undergo multiple cycles without discernable photodegradation.
\end{abstract}

Keywords: photolariat; azobenzene; macrocycles; receptor; photochromism

\section{Introduction}

A wealth of synthetic macrocyclic receptors have been reported over the last 3040 years, which have shown varying affinities to a wealth of guests, notably metal ions $(1,2)$. Among these receptors, stimulus-modulated species where the binding affinity can be modified as a response to a signal represents an expanding research area. This is due to the scope of potential applications in biomimetic nanotechnology as well as molecular biology, where liberation (or uptake) of a chemical effector at a given time is employed to ultimately allow elucidation of specific biological pathways $(3,4)$. In this context, photoactive receptors are particularly attractive as light energy can be applied to autonomous molecules in solution with high spatial and temporal resolution (5). Increasingly popular two photon absorption techniques offer a further possibility to irradiate with NIR light in the so-called therapeutic window, allowing deeper penetration of excitation light to activate deep lying molecules (6). Reversible photoinduced ion release and uptake imply photochromic receptors, where light switching between two distinct forms has a direct influence on binding. Indeed, a wealth of photochromes have been integrated with different receptors to give access to photocontrolled binding of a range of species $(7,8)$. The origin of the binding change is due to either electronic or steric effects. In the former case, on switching, electron density is less available to binding sites in one form with respect to the other, with a concomitant lowered binding constant. For the latter scenario, steric effects come into play when there is a significant geometrical change in the photoactive group between each form of the photochrome, which varies the shape of the receptor and hence suitability to bind a guest species. A popular choice of photochrome is azobenzene, which can be efficiently and reversibly switched from the thermally stable elongated trans-form to the more compact cis-form on UV irradiation. The return cis-to-trans transformation can be achieved thermally or by visible irradiation. In this way, different hosts such as cavitands and capsules can be rendered photoactive and more specifically the miniscule internal (yoctolitre) spaces available for chemical encapsulation can be controlled, which can ultimately lead to recognition, sequestration and reactivity of small molecules, depending on the host $(9,10)$. Among different manifestations of azobenzene in supramolecular hosts, one of the most successful implementations was the development of photocrowns where an azobenzene unit was directly incorporated in the macrocyclic host or in 'butterfly' variants $(7,11$ 15). However, binding constants are typically reasonably low and strategies to increase this parameter would be anticipated to widen the scope of implementation of these photoactive functional molecules. Concerning the photoswitching and photochemistry of these macrocylic azobenzene-containing hosts, the complexing ring imparts differing levels of strain on the azobenzene link, which as a 


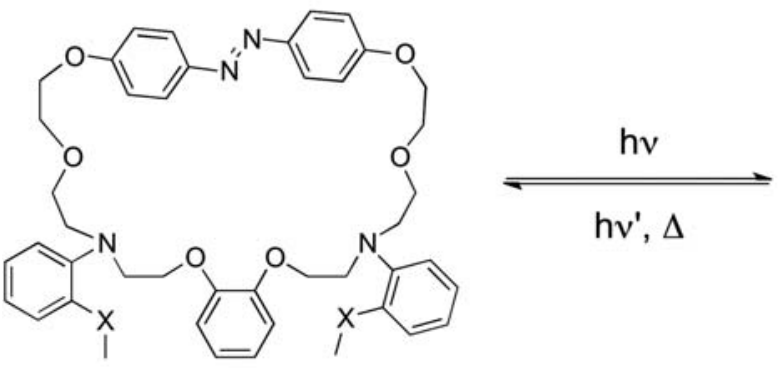

1a-E, 1b-E

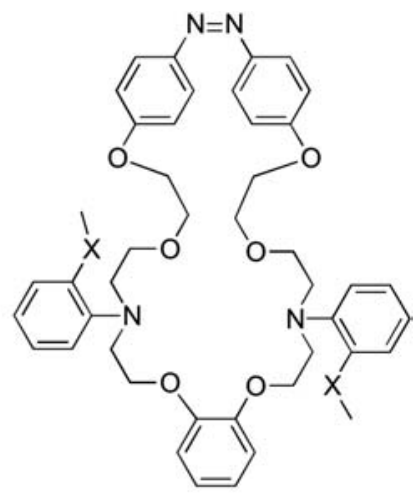

$1 a-Z, 1 b-Z$

Figure 1. Photoswitching of prototype photolariats $\mathbf{1 a}(X=O)$ and $\mathbf{1 b}(X=S)$. $E$ isomers should weakly complex with cations, while the $Z$ isomer is anticipated to be better adapted to complex.

function of size can drastically modify quantum yields, activation energy of cis-to-trans interconversion, absorption band intensities, as well as complexation selectivities (16 19).

As part of our ongoing work in the area of photocontrolled receptors and functional molecules and assemblies, we report herein the synthesis, photochemistry and complexation properties of lariat ethers, which incorporate a photoswitching azobenzene unit, deemed photolariats. The structural formulae of two members of this family are shown in Figure 1. In addition to the complexing heteroatom set of the macrocycle, two additional complexing atoms, oxygen and sulphur (1a and 1b, respectively), are added. Para- as opposed to orthosubstitution of the azobenzene with respect to the central nitrogens may be anticipated to amplify the geometrical difference between cis-form and trans-form, consequently changing the binding properties and photochromism.

\section{Experimental}

\subsection{Materials and methods}

Commercially available starting materials were obtained from Aldrich, AlfaAesar, Lancaster or Avocado. Compounds 2 (20) and 4 (21) were synthesised according to literature procedures. Anhydrous absolute ethanol and dimethylformamide (DMF) solvents were used as received. Dichloromethane and pyridine were distilled over calcium hydride $\left(\mathrm{CaH}_{2}\right)$ immediately before use. Tetrahydrofuran (THF) was dried over sodium/benzophenone and distilled immediately before use. Nuclear magnetic resonance (NMR): the ${ }^{1} \mathrm{H}$ and ${ }^{13} \mathrm{C}$ NMR spectra were recorded on a Bruker DPX200 $\left({ }^{1} \mathrm{H}\right.$ : $200 \mathrm{MHz})$ or a Bruker Avance $300\left({ }^{1} \mathrm{H}: 300 \mathrm{MHz} ;{ }^{13} \mathrm{C}\right.$ : $75 \mathrm{MHz})$ spectrometer. Chemical shifts $(\delta)$ are expressed relative to tetramethylsilane (TMS) using the residual signals of the deuterated solvents $\left(\mathrm{CDCl}_{3}, d_{6}\right.$-DMSO) as an internal reference. The coupling constants are calculated in Hertz $(\mathrm{Hz})$. For the assignment of signals, the following abbreviations are used: $\mathrm{s}$ singlet, $\mathrm{d}$ doublet, $\mathrm{t}$ triplet, q quartet, dd doublet of doublets and $\mathrm{m}$ multiplet. Mass spectrometry: the mass spectra were recorded by the Centre of Study and Structural Analysis Organic Molecules at the University of Bordeaux, France. Electrospray ionisation (ESI) mass spectra were performed on a QStar Elite mass spectrometer (Applied Biosystems). The instrument is equipped with an ESI source and spectra were recorded in the positive mode. The electrospray needle was maintained at $5000 \mathrm{~V}$ and operated at room temperature. Samples were introduced by injection through a $20 \mu \mathrm{L}$ sample loop into a $4500 \mu \mathrm{L} / \mathrm{min}$ flow of methanol from the LC pump. MALDIMS spectra were performed on a Voyager mass spectrometer (Applied Biosystems). The instrument is equipped with a pulsed $\mathrm{N}_{2}$ laser $(337 \mathrm{~nm}$ ) and a time-delayed extracted ion source. Spectra were recorded in the positive-ion mode using the reflectron and with an accelerating voltage of $20 \mathrm{kV}$. A dithranol matrix solution was employed. GC/MS mass spectra were performed on a Trace GC 2000 gas chromatograph equipped with a RTX5 MS column (length $15 \mathrm{~m}$; diameter $0.25 \mathrm{~mm}$; film $0.25 \mu \mathrm{m}$ ), coupled to a Thermofinnigan Trace electron impact (EI) mass spectrometer. Samples were measured using a source temperature of $200^{\circ} \mathrm{C}$ and a $150 \mu \mathrm{A}$ ionisation current. Electronic absorption spectroscopy: the electronic absorption spectra were recorded on optically dilute solutions in $1 \mathrm{~cm}$ pathlength quartz cells, whose temperature was thermostated, using a double beam Varian Cary 5000 UV vis-NIR spectrophotometer. Photoirradiation systems: irradiations at $365 \mathrm{~nm}$ were performed with a portable Fisher Bioblock mercury lamp (type thin layer chromatography (TLC)) with a power of $2 \times 6$ Watts. Isomerisation quantum yields were determined using stirred standard solutions irradiating at 334 or $365 \mathrm{~nm}$ with a mercury xenon lamp equipped with a monochromator. A ferrioxalate actinometer was employed following the Hatchard and Parker method (22). Photoisomerisation (trans-to-cis) of $\mathbf{1 a}$ and $\mathbf{1 b}$ was performed at $365 \mathrm{~nm}$ while parent azobenzene isomerisation was achieved at $334 \mathrm{~nm}$ because of the differences in the electronic 
absorption spectra. The appropriate actinometer quantum yield value was used in each case and correction was made for incomplete light absorption (22). All irradiation samples were air-equilibrated.

Single-crystal X-ray diffraction analysis: 1a: summary of data CCDC 864623: formula: $\mathrm{C}_{44} \mathrm{H}_{50} \mathrm{~N}_{4} \mathrm{O}_{8}$; unit cell parameters: $a \quad 14.5845 \quad(5), \quad b \quad 11.8355 \quad$ (4), c $22.7467(8), \beta \quad 97.576(2)$; space group C2/c; crystal data: $\left(\mathrm{C}_{44} \mathrm{H}_{50} \mathrm{~N}_{4} \mathrm{O}_{8}\right), M \quad 762.88, T$ 213(2) K, monoclinic, space group C2/c, $a \quad 14.5845$ (5), $b \quad 11.8355$ (4), c 22.7467 (8) A, $\beta \quad 97.576$ (2), $V \quad 3892.1$ (2) $\AA^{3}, \mathrm{Dc} \quad 1.302 \mathrm{~g} \mathrm{~cm}^{3}, Z \quad 4, F(000) \quad 1624,17,692$ reflections measured, 2195 independent reflections $\left(\begin{array}{ll}R_{\text {int }} & 0.049\end{array}\right)$, refinement on $F^{2}$ against all reflections. The weighted $R$-factor $\mathrm{wR}$ and goodness of fit GOF are based on $F^{2}$, GOF $1.09, R\left[F^{2}>2 \sigma\left(F^{2}\right)\right] \quad 0.085$, $\mathrm{wR}\left(F^{2}\right)$ 0.259. Hydrogen site location was inferred from neighbouring sites, $\mathrm{H}$-atom parameters constrained, 2195 reflections used for refinement, 254 parameters and no restraint. The data collection was performed on a Bruker microstar rotating anode at the $\mathrm{Cu} \mathrm{K} \alpha$ wavelength; cell refinement and data reduction were computed with the Bruker suite X8 Proteum; SHELXD97 (Sheldrick, 2008) was used to solve the crystal structure and SHELXL97 (Sheldrick, 1997) for refinement.

5a: Summary of data CCDC 864624: crystal data: $\left(\mathrm{C}_{24} \mathrm{H}_{24} \mathrm{~N}_{2} \mathrm{O}_{6}\right), M \quad 436.45, T 213$ (2) $\mathrm{K}$, orthorhombic, space group Pcca, $a 16.4963$ (8), b 17.9052 (9), c 7.1865 (4) A, $V \quad 2122.67(19) \AA^{3}, \mathrm{Dc} \quad 1.366 \mathrm{~g} \mathrm{~cm}^{3}$, $Z$ 4, $F(000)$ 920, 10,641 reflections measured, 949 independent reflections ( $\left.R_{\text {int }} 0.035\right)$, refinement on $F^{2}$ against all reflections. The weighted $R$-factor $\mathrm{wR}$ and goodness of fit GOF are based on $F^{2}$, GOF 1.33, $R\left[F^{2}>2 \sigma\left(F^{2}\right)\right] \quad 0.04, \operatorname{wR}\left(F^{2}\right) \quad 0.115$. Hydrogen site location was inferred from neighbouring sites, H-atom parameters constrained, 949 reflections used for refinement, 147 parameters and no restraint. The data collection has been performed on a Bruker microstar rotating anode at the $\mathrm{Cu} \mathrm{K} \alpha$ wavelength; cell refinement and data reduction were computed with the Bruker suite X8 Proteum; SHELXD97 (Sheldrick, 2008) was used to solve the crystal structure and SHELXL97 (Sheldrick, 1997) for refinement.

5b: Summary of data CCDC 864625; crystal data: $\left(\mathrm{C}_{24} \mathrm{H}_{24} \mathrm{~N}_{2} \mathrm{O}_{4} \mathrm{~S}_{2}\right), M \quad 468.57, T 213$ (2) $\mathrm{K}$, triclinic, space group P-1, $a \quad 4.702(6), b \quad 13.869(5), c \quad 18.607$ (8) A, $\alpha \quad 109.90^{\circ}, \beta \quad 90.06^{\circ}, \gamma \quad 99.33^{\circ}, V \quad 1123.8$ (2) $\AA^{3}$, Dc $1.385 \mathrm{~g} \mathrm{~cm}^{3}, Z$ 2, $F(000) \quad 492,3155$ reflections measured, 2340 independent reflections ( $R_{\text {int }} 0.223$ ), refinement on $F^{2}$ against all reflections. The weighted $R$-factor $\mathrm{wR}$ and goodness of fit GOF are based on $F^{2}$, GOF $0.656, \quad R\left[F^{2}>2 \sigma\left(F^{2}\right)\right] \quad 0.16$, $\mathrm{wR}\left(F^{2}\right) \quad$ 0.36. Hydrogen site location was inferred from neighbouring sites, H-atom parameters constrained, 5345 reflections used for refinement, 239 parameters and 17 restraints. Data collection was performed on a Rigaku MM07 rotating anode at the $\mathrm{Cu} \mathrm{K} \alpha$ wavelength. The CrystalClear (Rigaku/MSC Inc., 2006) suite was used for cell refinement and data reduction; SUPERFLIP (Palatinus, 2007) solved the structure that has been refined using SHELXL97 (Sheldrick, 1997); The WinGX (Farrugia, 1999) software was used to prepare material for publication. It has to be noted that it was not possible to collect better data on these tiny and X-ray sensitive crystals.

\subsection{Synthesis procedures}

Structural formulae of molecules $\mathbf{1 a} \mathbf{9 b}$ are shown in Figure 2.

3: To a solution of $2(657 \mathrm{mg}, 2.92 \mathrm{mmol})$ in DCM $(70 \mathrm{~mL})$ were added thionyl chloride $(1.52 \mathrm{~mL}, 20.9 \mathrm{mmol})$ and triethylamine $(0.42 \mathrm{~mL}, 3.0 \mathrm{mmol})$ at $0^{\circ} \mathrm{C}$. The mixture was refluxed for $2 \mathrm{~h}$. The reaction mixture was concentrated in vacuo and was used in coupling reactions with $\mathbf{6 a}$ or $\mathbf{6 b}$ without further purification.

5a: A mixture of $4(2.00 \mathrm{~g}, 8.85 \mathrm{mmol})$, thionyl chloride $(8.8 \mathrm{~mL})$ and triethylamine $(1.8 \mathrm{~mL})$ in DCM $(300 \mathrm{~mL})$ was refluxed for $3 \mathrm{~h}$. The solvent and excess thionyl chloride were removed in vacuo, yielding the diacid chloride as a light brown solid. To a well-stirred solution of this diacid chloride $(8.85 \mathrm{mmol})$ in DCM $(150 \mathrm{~mL})$, a solution of $o$-anisidine $(2.03 \mathrm{~mL}, 18 \mathrm{mmol})$ and triethylamine $(2.47 \mathrm{~mL}, 17.7 \mathrm{mmol})$ in DCM $(150 \mathrm{~mL})$ was added dropwise. The reaction mixture was stirred at room temperature for $6 \mathrm{~h}$ and was then washed with water $(3 \times 100 \mathrm{~mL})$ to remove the triethylammonium chloride and dried over $\mathrm{MgSO}_{4}$. The solvent was removed in vacuo and the residue was purified by column chromatography (silica, pentane/ethyl acetate, 7/3, v/v). The obtained product was further purified by recrystallisation from methanol, yielding 5a $(2.37 \mathrm{~g}, 61 \%) .{ }^{1} \mathrm{H} \mathrm{NMR}\left(\mathrm{CDCl}_{3}\right.$, $300 \mathrm{MHz}, \mathrm{TMS}): \delta 9.21(\mathrm{~s}, 2 \mathrm{H}, \mathrm{NH}) ; 8.43(\mathrm{~d}, J \quad 7.9 \mathrm{~Hz}$, $\left.2 \mathrm{H}, \mathrm{CH}_{A r}\right) ; 7.00\left(\mathrm{~m}, 8 \mathrm{H}, \mathrm{CH}_{A r}\right) ; 6.59$ (d, J $7.9 \mathrm{~Hz}, 2 \mathrm{H}$, $\left.\mathrm{CH}_{A r}\right) ; 4.72\left(\mathrm{~s}, 4 \mathrm{H}, \mathrm{CH}_{2}\right) ; 3.46\left(\mathrm{~s}, 6 \mathrm{H}, \mathrm{CH}_{3}\right) \mathrm{ppm} .{ }^{13} \mathrm{C}$ NMR $\left(\mathrm{CDCl}_{3}, 75 \mathrm{MHz}, \mathrm{TMS}\right): \delta 165.9 ; 148.3 ; 147.2$; $126.8 ; 124.5 ; 123.0 ; 120.9 ; 120.0 ; 114.2 ; 110.0 ; 68.5 ; 55.5$. GC/MS (EI): $m / z, 436.2\left(24 \%, \mathrm{M}^{+}\right), 122.9$ (100\%).

5b: The sulphur analogue $\mathbf{5 b}(9.74 \mathrm{~g}, 74 \%)$ was obtained similarly to 5a using 2-(methylthio)aniline. ${ }^{1} \mathrm{H}$ NMR $\left(\mathrm{CDCl}_{3}, 300 \mathrm{MHz}, \mathrm{TMS}\right): \delta 9.54(\mathrm{~s}, 2 \mathrm{H}, \mathrm{NH}) ; 8.34(\mathrm{~d}$, $\left.J \quad 8.2 \mathrm{~Hz}, 2 \mathrm{H}, \mathrm{CH}_{A r}\right) ; 7.37\left(\mathrm{~d}, J \quad 7.8 \mathrm{~Hz}, 2 \mathrm{H}, \mathrm{CH}_{A r}\right) ; 7.28$ (d, $\left.J \quad 8.0 \mathrm{~Hz}, 2 \mathrm{H}, \mathrm{CH}_{A r}\right) ; 7.07$ (d, J $\left.7.5 \mathrm{~Hz}, 2 \mathrm{H}, \mathrm{CH}_{A r}\right)$; $7.02\left(\mathrm{~s}, 4 \mathrm{H}, \mathrm{CH}_{A r}\right) ; 4,76\left(\mathrm{~s}, 4 \mathrm{H}, \mathrm{CH}_{2}\right) ; 2,14\left(\mathrm{~s}, 6 \mathrm{H}, \mathrm{CH}_{3}\right) \mathrm{ppm}$. ${ }^{13} \mathrm{C}$ NMR $\left(75 \mathrm{MHz}, \mathrm{CDCl}_{3}\right): \delta 166.6 ; 147.6 ; 137.7 ; 132.5$; $128.7 ; 126.6 ; 125.2 ; 123.4 ; 120.9 ; 114.9 ; 69.2 ; 18.5$ ppm. MS (ESI): $m / z 469.1\left(\mathrm{M}+\mathrm{H}^{+}\right) ; 491.1\left(\mathrm{M}+\mathrm{Na}^{+}\right)$.

6a: To a solution of diamide $\mathbf{5 a}(2.06 \mathrm{~g}, 4.73 \mathrm{mmol})$ in THF $(350 \mathrm{~mL})$, a solution of $\mathrm{BH}_{3}$ in THF $(1 \mathrm{M} ; 47 \mathrm{~mL}$, $47 \mathrm{mmol}$ ) was added dropwise. The mixture was refluxed for $65 \mathrm{~h}$, and the reaction was monitored by TLC (pentane/ethyl acetate, $7 / 3, v / v)$. Water $(10 \mathrm{~mL})$ was 

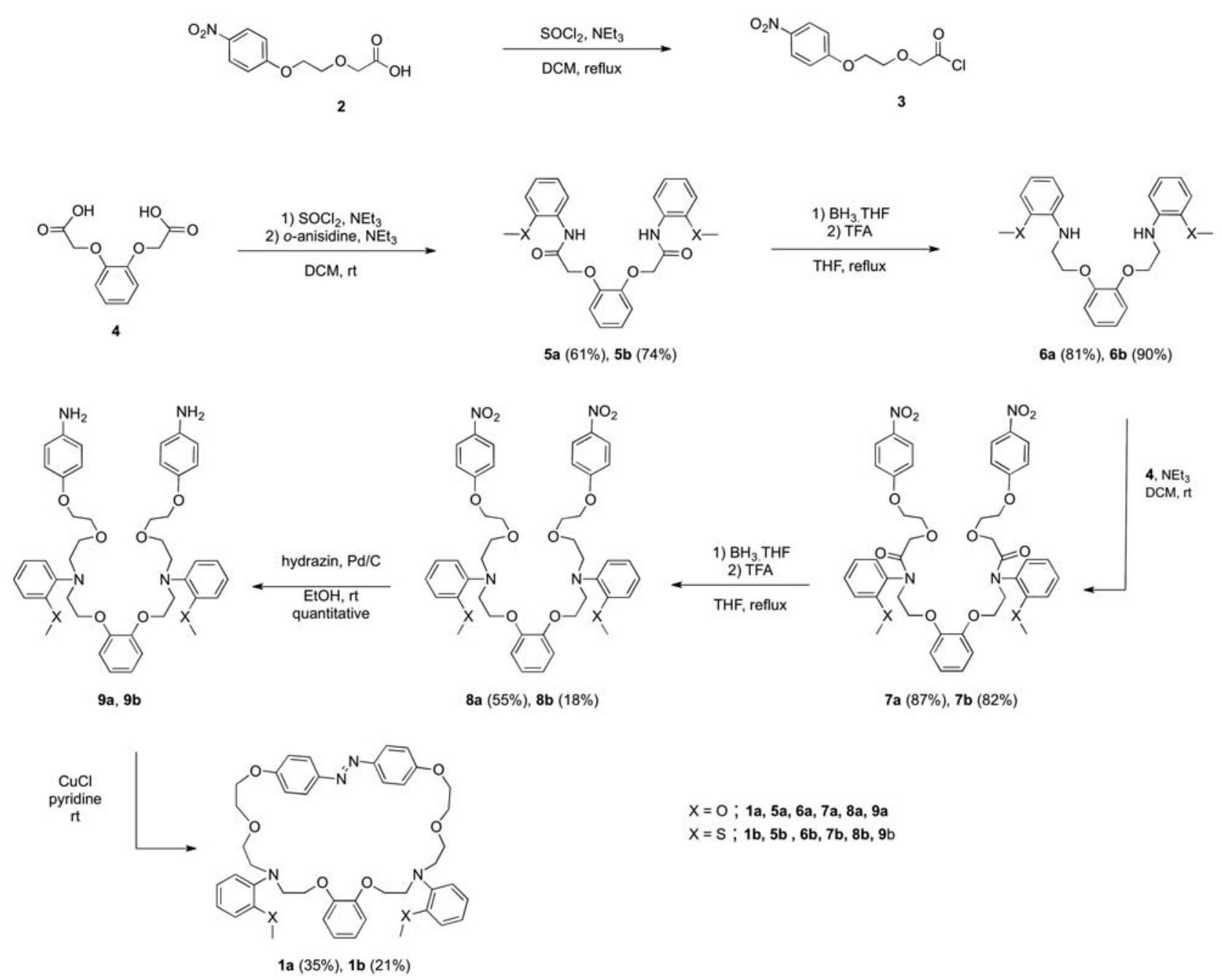

$X=0 ; 1 a, 5 a, 6 a, 7 a, 8 a, 9 a$

$X=S ; 1 b, 5 b, 6 b, 7 b, 8 b, 9 b$

Figure 2. Synthesis of photolariats $\mathbf{1 a}$ and $\mathbf{1 b}$.

added to slow down $\mathrm{H}_{2}$ evolution and solvents were evaporated in vacuo. The resulting white solid was used without further purification. The crude product was dissolved in THF (200 mL), and TFA (17.8 mL, $0.24 \mathrm{~mol})$ was added dropwise. The solution was then refluxed for $1 \mathrm{~h}$. THF was evaporated, and an aqueous solution of sodium hydroxide was added to neutralise the reaction mixture. After extraction with DCM, the organic phase was dried over $\mathrm{MgSO}_{4}$. The solvent was evaporated in vacuo, and the residue was purified by chromatography (silica gel, pentane/ethyl acetate, $8 / 2, v / v)$ to afford diamine $6 \mathbf{a}$ (1.56 g, 81\%) as a solid. ${ }^{1} \mathrm{H} \mathrm{NMR}\left(\mathrm{CDCl}_{3}, 300 \mathrm{MHz}, \mathrm{TMS}\right)$ : $\delta 6.66\left(\mathrm{~m}, 12 \mathrm{H}, \mathrm{CH}_{A r}\right) ; 4.78(\mathrm{~s}, 2 \mathrm{H}, \mathrm{NH}) ; 4.24(\mathrm{t}, 4 \mathrm{H}$, $\left.\mathrm{CH}_{2} \mathrm{O}\right) ; 3.78\left(\mathrm{~s}, 6 \mathrm{H}, \mathrm{CH}_{2} \quad \mathrm{O}\right) ; 3.57\left(\mathrm{t}, 4 \mathrm{H}, \mathrm{CH}_{2} \quad \mathrm{~N}\right) \mathrm{ppm}$. ${ }^{13} \mathrm{C} \mathrm{NMR}\left(\mathrm{CDCl}_{3}, 75 \mathrm{MHz}, \mathrm{TMS}\right): \delta 149.0 ; 147.1 ; 137.9$; $121.9 ; 121.2 ; 116.7 ; 115.3 ; 109.9 ; 109.5 ; 68.2 ; 55.3$; 43.0 ppm. GC/MS (EI): $m / z 408.2\left(\mathrm{M}^{+}\right) ; 135.9$.

6b: The sulphur analogue $6 \mathbf{b}(1.96 \mathrm{~g}, 90 \%)$ was obtained similarly to $\mathbf{6 a}$ using $\mathbf{5 b} .{ }^{1} \mathrm{H} \mathrm{NMR}\left(\mathrm{CDCl}_{3}\right.$, $300 \mathrm{MHz}, \mathrm{TMS}): \delta 7.36\left(\mathrm{~d}, J \quad 8.1 \mathrm{~Hz}, 2 \mathrm{H}, \mathrm{CH}_{A r}\right) ; 7.16(\mathrm{t}$,
J $\left.7.5 \mathrm{~Hz}, 2 \mathrm{H}, \mathrm{CH}_{A r}\right) ; 6.92\left(\mathrm{~m}, 4 \mathrm{H}, \mathrm{CH}_{A r}\right) ; 6.626 .67(\mathrm{~m}$, $\left.4 \mathrm{H}, \mathrm{CH}_{A r}\right) ; 5.37(\mathrm{~s}, 2 \mathrm{H}, \mathrm{NH}) ; 4.24(\mathrm{t}, J \quad 5.7 \mathrm{~Hz}, 4 \mathrm{H}$, $\left.\mathrm{CH}_{2} \mathrm{O}\right) ; 3.59\left(\mathrm{t}, \mathrm{J} \quad 5.9 \mathrm{~Hz}, 4 \mathrm{H}, \mathrm{CH}_{2} \mathrm{~N}\right) ; 2.25\left(\mathrm{~s}, 6 \mathrm{H}, \mathrm{CH}_{3}\right)$ ppm. ${ }^{13} \mathrm{C}$ NMR $\left(75 \mathrm{MHz}, \mathrm{CDCl}_{3}\right): \delta 149.2 ; 148.0 ; 134.0$; $129.4 ; 122.2 ; 120.7 ; 117.8 ; 115.4 ; 110.4 ; 68.2 ; 43.5$; $18.1 \mathrm{ppm}$. MS (ESI): $m / z 441.1675\left(\mathrm{M}+\mathrm{H}^{+}\right) ; 463.1491$ $\left(\mathrm{M}+\mathrm{Na}^{+}\right)$; calculated $\mathrm{C}_{24} \mathrm{H}_{28} \mathrm{~N}_{2} \mathrm{O}_{2} \mathrm{~S}_{2} \mathrm{Na}: 463.1484$.

7a: To a solution of $\mathbf{3}(0.76 \mathrm{~g}, 2.92 \mathrm{mmol}, 4 \mathrm{eq}$.$) in$ DCM $(50 \mathrm{~mL})$, triethylamine $(0.4 \mathrm{~mL})$ was added dropwise at $0^{\circ} \mathrm{C}$ and $7 \mathbf{a}(0.27 \mathrm{~g}, 0.65 \mathrm{mmol}, 1$ eq. $)$ was subsequently introduced dropwise at room temperature. This mixture was stirred for $22 \mathrm{~h}$, diluted in DCM and washed with a saturated aqueous solution of $\mathrm{NaCl}$ $(200 \mathrm{~mL})$ and water $(200 \mathrm{~mL})$. The crude product was purified by chromatography (silica gel, ethyl acetate/pentane, 7/3, v/v) yielding 7a (487 mg, 87\%). ${ }^{1} \mathrm{H}$ NMR $\left(\mathrm{CDCl}_{3}, 300 \mathrm{MHz}, \mathrm{TMS}\right): \delta 8.16(\mathrm{~d}, J 9.3 \mathrm{~Hz}, 4 \mathrm{H}$, $\left.\mathrm{CH}_{A r}\right) ; 7.357 .23\left(\mathrm{~m}, 4 \mathrm{H}, \mathrm{CH}_{A r}\right) ; 6.94\left(\mathrm{~m}, 4 \mathrm{H}, \mathrm{CH}_{A r}\right)$; $6.91\left(\mathrm{~d}, J \quad 9.3 \mathrm{~Hz}, 4 \mathrm{H}, \mathrm{CH}_{A r}\right) ; 6.84\left(\mathrm{~m}, 4 \mathrm{H}, \mathrm{CH}_{A r}\right) ; 4.22$ $3.86\left(\mathrm{~m}, 20 \mathrm{H}, \mathrm{CH}_{2}\right) ; 3.77\left(\mathrm{~s}, 6 \mathrm{H}, \mathrm{CH}_{3}\right) \mathrm{ppm} .{ }^{13} \mathrm{C} \mathrm{NMR}$ 
$\left(\mathrm{CDCl}_{3}, 75 \mathrm{MHz}, \mathrm{TMS}\right): \delta 179.4 ; 170.1 ; 163.8 ; 155.1$; $148.3 ; 141.6 ; 130.1 ; 129.5 ; 125.9 ; 121.3 ; 114.6 ; 113.5$; $111.9 ; 69.5 ; 69.4 ; 68.4 ; 65.8 ; 65.7 ; 55.5 ; 48.4$ ppm. MS (ESI): $m / z 855.4\left(\mathrm{M}+\mathrm{H}^{+}\right) ; 877.4\left(\mathrm{M}+\mathrm{Na}^{+}\right)$.

7b: The sulphur analogue $\mathbf{7 b}(680 \mathrm{mg}, 82 \%)$ was obtained similarly to $7 \mathbf{a}$ using $\mathbf{6 b} .{ }^{1} \mathrm{H}$ NMR $\left(\mathrm{CDCl}_{3}, 200\right.$ MHz, TMS): $\delta 8.10\left(\mathrm{~d}, J \quad 9.0 \mathrm{~Hz}, 4 \mathrm{H}, \mathrm{CH}_{A r}\right) ; 7.207 .36$ $\left(\mathrm{m}, 4 \mathrm{H}, \mathrm{CH}_{A r}\right) ; 7.057 .17\left(\mathrm{~m}, 4 \mathrm{H}, \mathrm{C} H_{A r}\right) ; 6.88$ (d, J 9.2 Hz, 4H, CH $\left.H_{A r}\right) ; 6.82\left(\mathrm{~s}, 4 \mathrm{H}, \mathrm{CH}_{A r}\right) ; 3.38$ $4.47\left(\mathrm{~m}, 2 \mathrm{H}, \mathrm{CH}_{2}\right) ; 2.40\left(\mathrm{~s}, 6 \mathrm{H}, \mathrm{CH}_{3}\right) \mathrm{ppm} .{ }^{13} \mathrm{C} \mathrm{NMR}$ $\left(\mathrm{CDCl}_{3}, 75 \mathrm{MHz}, \mathrm{TMS}\right): \delta 169.8 ; 163.8 ; 148.3 ; 141.6$; $138.6 ; 137.2 ; 129.9 ; 129.6 ; 125.8 ; 125.4 ; 124.8 ; 121.5$; $114.6 ; 113.8 ; 69.6 ; 69.5 ; 68.3 ; 65.8 ; 47.5 ; 14.1$ ppm. MS (ESI): $m / z 909.2\left(\mathrm{M}+\mathrm{Na}^{+}\right)$.

8a: Method A. To a solution of $6 \mathbf{a}(0.20 \mathrm{~g}, 0.49 \mathrm{mmol})$ in DMF $(10 \mathrm{~mL}), \mathrm{Cs}_{2} \mathrm{CO}_{3}(0.80 \mathrm{~g}, 2.46 \mathrm{mmol})$ and 1-[2-(2iodoethoxy)ethoxy]-4-nitrobenzene (23) (0.81 g, $2.40 \mathrm{mmol})$ were added, and the reaction mixture was stirred for $20 \mathrm{~h}$ at $120^{\circ} \mathrm{C}$, after which time another aliquot of 1-[2-(2iodoethoxy)ethoxy]-4-nitrobenzene $(0.81 \mathrm{~g}, 2.40 \mathrm{mmol})$ was added. This was repeated after another $20 \mathrm{~h}$ of reaction, and the reaction mixture was stirred for an additional $60 \mathrm{~h}$ at $120^{\circ} \mathrm{C}$. The reaction mixture was allowed to cool to room temperature and was filtered over a pad of Celite. The solvent of the filtrate was removed in vacuo, and the residue was purified by chromatography (silica gel, pentane/ethyl acetate, $6 / 4, v / v)$ yielding $\mathbf{8 a}(52 \mathrm{mg}, 13 \%)$. Method $B$. To a solution of diamide 7a $(1.00 \mathrm{~g}, 1.17 \mathrm{mmol})$ in THF $(60 \mathrm{~mL})$, a solution of $\mathrm{BH}_{3}$ in THF (1 M; $\left.12 \mathrm{~mL}, 12 \mathrm{mmol}\right)$ was added dropwise. The mixture was refluxed for $15 \mathrm{~h}$, and the reaction was monitored with TLC (petroleum ether/ethyl acetate, 1/4, v/v). Some water was added to slow down $\mathrm{H}_{2}$ evolution and solvents were evaporated in vacuo. The resulting solid was used without further purification. The solid was dissolved in THF $(60 \mathrm{~mL})$, TFA (4 mL) was added dropwise and the mixture was refluxed for $1 \mathrm{~h}$. The solvent was removed in vacuo. The residue was dissolved in water, and the reaction mixture was neutralised with an aqueous solution of sodium hydroxide. After extraction with DCM, the organic phase was washed with water and was dried over $\mathrm{MgSO}_{4}$. The solvent was evaporated in vacuo, and the residue was purified by chromatography (silica gel, petroleum ether/ethyl acetate, $3 / 2, v / v)$ affording diamine 8a $(0.53 \mathrm{~g}, 55 \%) .{ }^{1} \mathrm{H}$ NMR $\left(\mathrm{CDCl}_{3}, 400 \mathrm{MHz}, \mathrm{TMS}\right): \delta 8.14\left(\mathrm{~d}, J \quad 9.24 \mathrm{~Hz}, 4 \mathrm{H}, \mathrm{CH}_{A r}\right)$; 7.07 (d, $\left.J \quad 7.88 \mathrm{~Hz}, 2 \mathrm{H}, \mathrm{CH}_{A r}\right) ; 6.97$ (t, $J \quad 8.0 \mathrm{~Hz}, 2 \mathrm{H}$, $\left.\mathrm{CH}_{A r}\right) ; 6.90\left(\mathrm{~d}, J \quad 9.28 \mathrm{~Hz}, 4 \mathrm{H}, \mathrm{CH}_{A r}\right) ; 6.85(\mathrm{~d}, J \quad 7.72 \mathrm{~Hz}$, $\left.4 \mathrm{H}, \mathrm{CH}_{A r}\right) ; 6.80\left(\mathrm{~m}, 4 \mathrm{H}, \mathrm{CH}_{A r}\right) ; 4.09\left(\mathrm{t}, J \quad 5 \mathrm{~Hz}, 4 \mathrm{H}, \mathrm{CH}_{2}\right)$; $4.05\left(\mathrm{t}, J \quad 6.68 \mathrm{~Hz}, 4 \mathrm{H}, \mathrm{CH}_{2}\right) ; 3.82\left(\mathrm{~s}, 6 \mathrm{H}, \mathrm{CH}_{3}\right) ; 3.76(\mathrm{t}$, $\left.J \quad 4.8 \mathrm{~Hz}, 4 \mathrm{H}, \mathrm{CH}_{2}\right) ; 3.64\left(\mathrm{t}, J \quad 5.72 \mathrm{~Hz}, 4 \mathrm{H}, \mathrm{CH}_{2}\right) ; 3.62(\mathrm{t}$, $\left.J \quad 6.32 \mathrm{~Hz}, 4 \mathrm{H}, \mathrm{CH}_{2}\right) ; 3.50\left(\mathrm{t}, J \quad 5.92 \mathrm{~Hz}, 4 \mathrm{H}, \mathrm{CH}_{2}\right) \mathrm{ppm}$. ${ }^{13} \mathrm{C} \mathrm{NMR}\left(\mathrm{CDCl}_{3}, 75 \mathrm{MHz}, \mathrm{TMS}\right): \delta 163.8 ; 153.5 ; 148.7$; $141.4 ; 138.7 ; 125.7 ; 123.2 ; 122.0 ; 121.0 ; 120.7 ; 114.5 ; 113.6$; $111.9 ; 69.8 ; 68.9 ; 68.1 ; 67.2 ; 55.4 ; 52.8 ; 52.4$ ppm. MS (MALDI): $m / z 826.2\left(\mathrm{M}^{+}\right), 849.3\left(\mathrm{M}+\mathrm{Na}^{+}\right)$. 8b: The sulphur analogue $\mathbf{8 b}(0.14 \mathrm{~g}, 18 \%)$ was obtained similarly to $\mathbf{8 a}$ (Method B) using $\mathbf{7 b}$. ${ }^{1} \mathrm{H}$ NMR $\left(\mathrm{CDCl}_{3}, 300 \mathrm{MHz}, \mathrm{TMS}\right): \delta 8.14(\mathrm{~d}, J 9.2 \mathrm{~Hz}, 4 \mathrm{H}$, $\left.\mathrm{CH}_{A r}\right) ; 7.22\left(\mathrm{~d}, J \quad 8.1 \mathrm{~Hz}, 2 \mathrm{H}, \mathrm{CH}_{A r}\right) ; 7.027 .11(\mathrm{~m}, 6 \mathrm{H}$, $\left.\mathrm{CH}_{A r}\right) ; 6.90\left(\mathrm{~d}, J \quad 9.6 \mathrm{~Hz}, 4 \mathrm{H}, \mathrm{CH}_{A r}\right) ; 6.81\left(\mathrm{~s}, 4 \mathrm{H}, \mathrm{CH}_{A r}\right)$; $4.11\left(\mathrm{t}, J \quad 4.5 \mathrm{~Hz}, 4 \mathrm{H}, \mathrm{CH}_{2}\right) ; 4.02(\mathrm{t}, J \quad 6.6 \mathrm{~Hz}, 4 \mathrm{H}$, $\left.\mathrm{CH}_{2}\right) ; 3.76\left(\mathrm{t}, \mathrm{J} \quad 4.5 \mathrm{~Hz}, 4 \mathrm{H}, \mathrm{CH}_{2}\right) ; 3.61(\mathrm{t}, \mathrm{J} \quad 6.3 \mathrm{~Hz}$, $\left.4 \mathrm{H}, \mathrm{CH}_{2}\right) ; 3.51\left(\mathrm{t}, \mathrm{J} 6.6 \mathrm{~Hz}, 4 \mathrm{H}, \mathrm{CH}_{2}\right) ; 3.41(\mathrm{t}$, J $\left.\quad 6.0 \mathrm{~Hz}, 4 \mathrm{H}, \mathrm{CH}_{2}\right) ; 2.35\left(\mathrm{~s}, 6 \mathrm{H}, \mathrm{CH}_{3}\right) \mathrm{ppm} .{ }^{13} \mathrm{C} \mathrm{NMR}$ $\left(\mathrm{CDCl}_{3}, 75 \mathrm{MHz}, \mathrm{TMS}\right): \delta 163.9 ; 148.7 ; 147.1 ; 141.6$; $137.8 ; 125.8 ; 125.4 ; 124.5 ; 124.2 ; 123.8 ; 121.1 ; 114.6$; $113.8 ; 70.0 ; 69.0 ; 68.2 ; 67.3 ; 53.9 ; 53.4 ; 14.2$ ppm. HRMS (ESI): $m / z 881.2832\left(\mathrm{M}+\mathrm{Na}^{+}\right)$; calculated $\mathrm{C}_{44} \mathrm{H}_{50} \mathrm{~N}_{4^{-}}$ $\mathrm{O}_{10} \mathrm{~S}_{2} \mathrm{Na}: 881.2860$.

9a: To a suspension of $10 \%$ palladium on charcoal $(53 \mathrm{mg})$ in ethanol $(15 \mathrm{~mL}), \mathbf{8 a}(200 \mathrm{mg}, 0.24 \mathrm{mmol})$ and hydrazine hydrate $(1.5 \mathrm{~mL}, 30.9 \mathrm{mmol})$ were added. The mixture was refluxed for $15 \mathrm{~h}$ and monitored by TLC (AcOEt/petroleum ether, 3/2, v/v). The solution was filtered hot over a pad of Celite, and the filtrate was evaporated in vacuo. The reaction is quantitative and further purification proved unnecessary. ${ }^{1} \mathrm{H} \mathrm{NMR}\left(\mathrm{CDCl}_{3}\right.$, $300 \mathrm{MHz}, \mathrm{TMS}): \delta 7.08\left(\mathrm{~d}, J \quad 7.8 \mathrm{~Hz}, 2 \mathrm{H}, \mathrm{CH}_{A r}\right) ; 6.97(\mathrm{t}$, $\left.J \quad 7.8 \mathrm{~Hz}, 2 \mathrm{H}, \mathrm{CH}_{A r}\right) ; 6.85\left(\mathrm{t}, J \quad 7.8 \mathrm{~Hz}, 4 \mathrm{H}, \mathrm{CH}_{A r}\right)$; $6.80\left(\mathrm{~s}, 4 \mathrm{H}, \mathrm{CH}_{A r}\right) ; 6.71\left(\mathrm{~d}, J \quad 9 \mathrm{~Hz}, 4 \mathrm{H}, \mathrm{CH}_{A r}\right) ; 6.59(\mathrm{~d}$, $\left.J \quad 9 \mathrm{~Hz}, 4 \mathrm{H}, \mathrm{CH}_{A r}\right) ; 4.05\left(\mathrm{t}, J \quad 6.9 \mathrm{~Hz}, 4 \mathrm{H}, \mathrm{CH}_{2}\right) ; 3.95$ (t, J $\left.5.1 \mathrm{~Hz}, 4 \mathrm{H}, \mathrm{CH}_{2}\right) ; 3.81\left(\mathrm{~s}, 6 \mathrm{H}, \mathrm{CH}_{3}\right) ; 3.69(\mathrm{t}$, J $\left.5.1 \mathrm{~Hz}, 4 \mathrm{H}, \mathrm{CH}_{2}\right) ; 3.65\left(\mathrm{~m}, 8 \mathrm{H}, \mathrm{CH}_{2}\right) ; 3.49(\mathrm{t}$, J $\left.\quad 5.7 \mathrm{~Hz}, 4 \mathrm{H}, \mathrm{CH}_{2}\right)$ ppm. ${ }^{13} \mathrm{C} \mathrm{NMR}\left(\mathrm{CDCl}_{3}, 75 \mathrm{MHz}\right.$, TMS): $\delta 153.5 ; 151.9 ; 148.7 ; 140.1 ; 138.8 ; 123.1 ; 122.0$; $121.0 ; 120.8 ; 116.4 ; 115.8 ; 113.7 ; 111.9 ; 69.6 ; 69.5 ; 68.0$; 67.1; 55.4; 52.8; 52.3 ppm. MS (ESI): $\mathrm{m} / \mathrm{z} 767.4$ $\left(\mathrm{M}+\mathrm{H}^{+}\right) ; 789.4\left(\mathrm{M}+\mathrm{Na}^{+}\right)$.

9b: Method A. The sulphur analogue $9 b(120 \mathrm{mg})$ was obtained analogously to $9 \mathbf{a}$ using $\mathbf{8 b}$. Method $B$. To a solution of diamide $\mathbf{7 b}(215 \mathrm{mg}, 0.25 \mathrm{mmol})$ in THF $(20 \mathrm{~mL})$, a solution of $\mathrm{BH}_{3}$ in THF $(1 \mathrm{M} ; 2.4 \mathrm{~mL}$, $2.4 \mathrm{mmol}$ ) was added dropwise. The mixture was refluxed for $15 \mathrm{~h}$, after which $20 \mathrm{~mL} \mathrm{MeOH}$ was added. The mixture was further refluxed for $1 \mathrm{~h}$ after which the solvent was removed in vacuo. The resulting solid was redissolved in $50 \mathrm{~mL} \mathrm{EtOH}, 10 \% \mathrm{Pd} / \mathrm{C}(100 \mathrm{mg})$ and $2.5 \mathrm{~mL}$ hydrazine hydrate $(48.5 \mathrm{mmol})$ were added and the suspension was refluxed for $6 \mathrm{~h}$. The suspension was filtered hot over a pad of Celite. The solvent and excess hydrazine of the filtrate were removed in vacuo, and the residue was purified by chromatography (silica gel, ethyl acetate/petroleum ether, 4/1, v/v), yielding 9b (140 mg, 75\%). ${ }^{1} \mathrm{H} \mathrm{NMR}\left(\mathrm{CDCl}_{3}\right.$, $300 \mathrm{MHz}, \mathrm{TMS}): \delta 7.26\left(\mathrm{~d}, J \quad 8.0 \mathrm{~Hz}, 2 \mathrm{H}, \mathrm{CH}_{A r}\right) ; 7.11$ $\left(\mathrm{m}, 6 \mathrm{H}, \mathrm{CH}_{A r}\right) ; 6.85\left(\mathrm{~s}, 4 \mathrm{H}, \mathrm{CH}_{A r}\right) ; 6.75(\mathrm{~d}, J \quad 8.8 \mathrm{~Hz}$, $\left.4 \mathrm{H}, \mathrm{CH}_{A r}\right) ; 6.63\left(\mathrm{~d}, J \quad 8.8 \mathrm{~Hz}, 4 \mathrm{H}, \mathrm{CH}_{A r}\right) ; 4.05(\mathrm{t}$, $\left.J \quad 6.7 \mathrm{~Hz}, 4 \mathrm{H}, \mathrm{CH}_{2}\right) ; 3.99\left(\mathrm{t}, J \quad 5.0 \mathrm{~Hz}, 4 \mathrm{H}, \mathrm{CH}_{2}\right) ; 3.73$ (t, $\left.J \quad 5.0 \mathrm{~Hz}, 4 \mathrm{H}, \mathrm{CH}_{2}\right) ; 3.63\left(\mathrm{t}, \mathrm{J} \quad 6.3 \mathrm{~Hz}, 4 \mathrm{H}, \mathrm{CH}_{2}\right.$ ); $3.56\left(\mathrm{t}, J \quad 6.57 \mathrm{~Hz}, 4 \mathrm{H}, \mathrm{CH}_{2}\right) ; 3.44(\mathrm{t}, J \quad 6.1 \mathrm{~Hz}, 4 \mathrm{H}$, $\left.\mathrm{CH}_{2}\right) ; 2.39$ (s, $\left.6 \mathrm{H}, \mathrm{CH}_{3}\right) \mathrm{ppm} .{ }^{13} \mathrm{C} \mathrm{NMR}\left(\mathrm{CDCl}_{3}, 75 \mathrm{MHz}\right.$, 
TMS): $\delta 152.0 ; 148.8 ; 147.3 ; 140.1 ; 137.8 ; 125.2 ; 124.6$; $124.3 ; 123.9 ; 121.1 ; 116.4 ; 115.9 ; 113.9 ; 69.8 ; 69.6 ; 68.1$; $67.3 ; 53.8 ; 53.4 ; 14.3$ ppm. HRMS (ESI): $\mathrm{m} / z$. 823.3531 $\left(\mathrm{M}+\mathrm{Na}^{+}\right)$; calculated $\mathrm{C}_{44} \mathrm{H}_{56} \mathrm{~N}_{4} \mathrm{O}_{6} \mathrm{~S}_{2} \mathrm{Na}: 823.3533$.

1a: A pyridine solution $(60 \mathrm{~mL})$ of dianiline $9 \mathbf{a}(66 \mathrm{mg}$, $0.086 \mathrm{mmol})$ and $\mathrm{CuCl}(65 \mathrm{mg}, 0.66 \mathrm{mmol})$ was stirred in the dark under air for $20 \mathrm{~h}$ at room temperature, and the solvent was then evaporated. The residue was purified by chromatography (silica gel, petroleum ether/ethyl acetate $1: 1, v / v)$ affording azolariat 1a $(23 \mathrm{mg}, 35 \%) .{ }^{1} \mathrm{H}$ NMR $\left(\mathrm{CDCl}_{3}, 300 \mathrm{MHz}, \mathrm{TMS}\right): \delta 7.75(\mathrm{~d}, J \quad 9 \mathrm{~Hz}, 4 \mathrm{H}$, $\left.\mathrm{CH}_{A r}\right) ; 7.12\left(\mathrm{~d}, J \quad 9 \mathrm{~Hz}, 4 \mathrm{H}, \mathrm{CH}_{A r}\right) ; 6.93\left(\mathrm{~m}, 4 \mathrm{H}, \mathrm{CH}_{A r}\right)$; $6.676 .85\left(\mathrm{~m}, 8 \mathrm{H}, \mathrm{CH}_{A r}\right) ; 4.37\left(\mathrm{t}, \mathrm{J} \quad 3.9 \mathrm{~Hz}, 4 \mathrm{H}, \mathrm{CH}_{2}\right)$; $3.81\left(\mathrm{t}, \mathrm{J} \quad 8.4 \mathrm{~Hz}, 4 \mathrm{H}, \mathrm{CH}_{2}\right) ; 3.78\left(\mathrm{~s}, 6 \mathrm{H}, \mathrm{CH}_{3} \mathrm{O}\right) ; 3.70$ (t, $\left.J \quad 4.2 \mathrm{~Hz}, 4 \mathrm{H}, \mathrm{CH}_{2}\right) ; 3.39\left(\mathrm{t}, J \quad 6 \mathrm{~Hz}, 4 \mathrm{H}, \mathrm{CH}_{2}\right) ; 3.28$ (t, $\left.J \quad 7.5 \mathrm{~Hz}, 4 \mathrm{H}, \mathrm{CH}_{2}\right) ; 3.14\left(\mathrm{t}, J \quad 5.7 \mathrm{~Hz}, 4 \mathrm{H}, \mathrm{CH}_{2}\right)$ ppm. ${ }^{13} \mathrm{C} \mathrm{NMR}\left(\mathrm{CDCl}_{3}, 75 \mathrm{MHz}, \mathrm{TMS}\right): \delta 161.1 ; 153.9$; $148.5 ; 147.3 ; 138.6 ; 124.2 ; 123.4 ; 122.2 ; 120.8 ; 116.4$; $114.7 ; 113.3 ; 112.0 ; 71.0 ; 69.7 ; 68.2 ; 66.3 ; 55.5 ; 52.9$; $29.8 \mathrm{ppm}$. HRMS (ESI): $m / z \quad 763.3711\left(\mathrm{M}+\mathrm{H}^{+}\right)$; $785.3527\left(\mathrm{M}+\mathrm{Na}^{+}\right)$; calculated $\mathrm{C}_{44} \mathrm{H}_{51} \mathrm{~N}_{4} \mathrm{O}_{8}$ : 763.3701.

1b: The sulphur analogue $\mathbf{1 b}(32 \mathrm{mg}, 21 \%)$ was obtained analogously to $\mathbf{1 a}$ using $\mathbf{9 b} .{ }^{1} \mathrm{H}$ NMR $\left(\mathrm{CDCl}_{3}\right.$, $300 \mathrm{MHz}, \mathrm{TMS}): \delta 7.77\left(\mathrm{~d}, J \quad 8.7 \mathrm{~Hz}, 4 \mathrm{H}, \mathrm{CH}_{A r}\right) ; 7.12$ $\left(\mathrm{d}, J \quad 9 \mathrm{~Hz}, 4 \mathrm{H}, \mathrm{CH}_{A r}\right) ; 7.07 .10\left(\mathrm{~m}, 8 \mathrm{H}, \mathrm{CH}_{A r}\right) ; 6.68$ $6.79\left(\mathrm{~m}, 4 \mathrm{H}, \mathrm{CH}_{A r}\right) ; 4.37\left(\mathrm{t}, \mathrm{J} \quad 3.9 \mathrm{~Hz}, 4 \mathrm{H}, \mathrm{CH}_{2}\right) ; 3.82(\mathrm{t}$, J $\left.\quad 7.5 \mathrm{~Hz}, 4 \mathrm{H}, \mathrm{CH}_{2}\right) ; 3.70\left(\mathrm{t}, \mathrm{J} \quad 3.9 \mathrm{~Hz}, 4 \mathrm{H}, \mathrm{CH}_{2}\right) ; 3.33$ (t, $\left.J \quad 6.3 \mathrm{~Hz}, 4 \mathrm{H}, \mathrm{CH}_{2}\right) ; 3.16\left(\mathrm{t}, J \quad 8.1 \mathrm{~Hz}, 4 \mathrm{H}, \mathrm{CH}_{2}\right)$; $2.99\left(\mathrm{t}, \mathrm{J} \quad 6 \mathrm{~Hz}, 4 \mathrm{H}, \mathrm{CH}_{2}\right) ; 2.33\left(\mathrm{~s}, 6 \mathrm{H}, \mathrm{CH}_{3}\right) \mathrm{ppm} .{ }^{13} \mathrm{C}$ NMR $\left(\mathrm{CDCl}_{3}, 75 \mathrm{MHz}, \mathrm{TMS}\right): \delta 161.1 ; 148.4 ; 147.3$; $147.0 ; 138.3 ; 125.5 ; 124.5 ; 124.1 ; 124.0 ; 123.7 ; 120.7$; $116.3 ; 113.4 ; 70.8 ; 69.5 ; 68.1 ; 66.3 ; 54.0 ; 54.0 ; 14.2 \mathrm{ppm}$. HRMS (ESI): $\mathrm{m} / \mathrm{z} 817.3059\left(\mathrm{M}+\mathrm{Na}^{+}\right)$; calculated $\mathrm{C}_{44} \mathrm{H}_{50} \mathrm{~N}_{4} \mathrm{O}_{6} \mathrm{NaS}_{2}: 817.3064\left(\mathrm{M}+\mathrm{Na}^{+}\right)$.

\section{Results and discussion}

\subsection{Synthesis and single-crystal $X$-ray diffraction structures}

Photolariats 1a and 1b were synthesised via a multi-step approach from a common precursor (4), as detailed in the preceding section and is schematised in Figure 2.

The diacid 4 was activated by thionyl chloride and was then reacted with an excess of either $o$-anisidine or (methylthio)aniline, yielding the corresponding amides 5a and $\mathbf{5 b}$ in moderate yields. Subsequently, the amides were reduced to secondary amines. The application of $\mathrm{LiAlH}_{4}$ as a reducing agent leads to decomposition, whereas $\mathrm{NaBH}_{4}$ was not capable of reducing the amides to any considerate extent. However, the application of borane in THF, followed by acid treatment to break the $\mathrm{N}$ B adduct bond, led to the efficient reduction in the amides of $\mathbf{5 a}$ and $\mathbf{5 b}$ to the corresponding amines $6 \mathbf{a}$ and $\mathbf{6 b}$ in good to excellent yields.

The direct alkylation of the secondary amines was discovered to be low yielding and slow even at elevated temperatures (8a could be isolated in $13 \%$ yield from $\mathbf{6 a}$ after heating at $120^{\circ} \mathrm{C}$ for $100 \mathrm{~h}$ ). Therefore, an alternative route via the secondary amides $7 \mathbf{a}$ and $\mathbf{7 b}$ was followed. The secondary amines $\mathbf{6} \mathbf{a}$ and $\mathbf{6} \mathbf{b}$ were reacted with acid chloride $\mathbf{3}$, yielding the corresponding amides $\mathbf{7 a}$ and $\mathbf{7 b}$ in good yields. Subsequently, the amides were reduced by borane in THF. In the case of the reduction in $\mathbf{7 b}$, this reaction proved ineffective. It was observed that in this case partial oxidation of the nitrobenzene moieties occurred.

Although it is known from literature reports that nitrobenzenes can be directly reduced to the corresponding symmetrical azobenzenes by $\mathrm{LiAlH}_{4}(24)$, it was, in light of the decomposition caused by this reagent in a previous step, decided to perform the azobenzene formation in two steps via the aniline intermediates. The reduction in the nitrobenzenes $\mathbf{8 a}$ and $\mathbf{8 b}$ to give anilines $\mathbf{9 a}$ and $\mathbf{9 b}$ proceeded quantitatively in both cases (25).

Inspired by a literature report of a one-pot borane reduction in a secondary amide and a subsequent $\mathrm{Pd} / \mathrm{C}$ catalysed hydrogenation (26), the direct multi-stage onepot reduction in amide $\mathbf{7 b}$ to aniline $\mathbf{9 b}$ was performed. The reaction proceeded in an improved overall yield of $75 \%$. Finally, the oxidation in anilines $\mathbf{9 a}$ and $\mathbf{9 b}$ to the corresponding azobenzenes $\mathbf{1 a}$ and $\mathbf{1 b}$ was achieved using a copper catalyst, prepared in situ from pyridine and $\mathrm{CuCl}$ (27). In this reaction, the thermodynamically favoured trans-isomers $(E)$ are exclusively formed.

Single-crystal X-ray diffraction data were also sought for the photolariats and some intermediates (see Figure 3(a) (c) and Experimental section). The crystal structure of $\mathbf{1 a}-\boldsymbol{E}$ (Figure 3 a) shows a $C_{2}$-symmetry axis. The trans-azobenzene moiety is almost planar, inducing a stretched conformation of the macrocycle. Both methoxybenzene groups are repulsed to the outside. The width of the cavity is around $3.9 \AA$, and the stretched conformation leads us to conclude that complexation of a

(a)

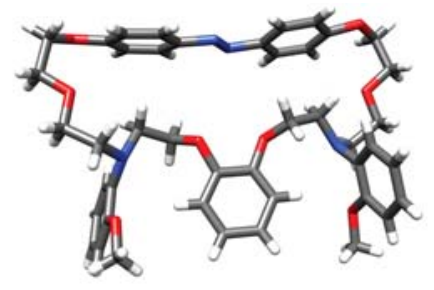

(b)

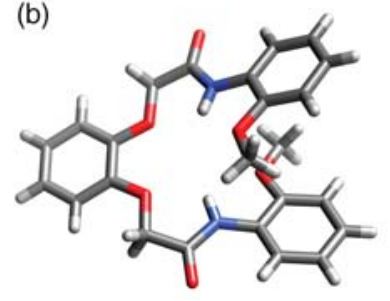

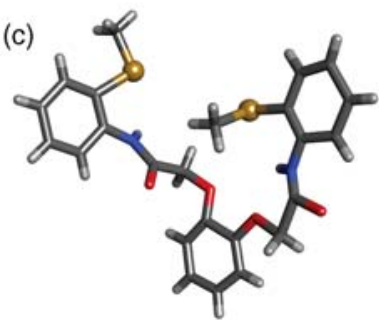

Figure 3. Single crystal X ray structure of $\mathbf{1 a} \boldsymbol{E}(\mathrm{a}), \mathbf{5 a}(\mathrm{b})$ and $\mathbf{5 b}(\mathrm{c})$. Sulphur atoms are represented as spheres. 
cation inside this cavity should be disfavoured. On the other hand, the less elongated $c i$ s-form may be anticipated to have a less drastic effect on the binding site. Indeed, molecular modelling suggests that $\mathbf{1 a}-\boldsymbol{Z}$ adopts a more globular shape, which is conducive with complexation. (Due to the low energy barrier for single $\mathrm{C} C$ bond rotation, one definitive structural orientation is not shown.)

Additionally, intermediates $\mathbf{5 a}$ and $\mathbf{5 b}$ were crystallised, and the structures obtained from single crystals grown in methanol are shown in Figure 3(b),(c), respectively. Their X-ray structures are very different, despite the similarity of their compositions. In 5a, the intramolecular hydrogen bonds formed (implicating the methoxy oxygen and the relatively acidic amide proton) facilitates folding of the molecule, which tends to a helical structure. In contrast in $\mathbf{5 b}$, intramolecular hydrogen bonds are less favoured and only intermolecular interactions were identified. This underlies the difference between oxygen and sulphur atoms as hydrogen bond acceptors.

\subsection{Photo- and thermal isomerisation}

As trans-conformations of azobenzenes in general are thermodynamically more stable than cis-conformations, it is anticipated that at equilibrium in the dark the population of photolariats will consist of $100 \%$ trans-form (1-E). Figure 4 shows this to be the case for both 1a (Figure 4(a), solid line) and $\mathbf{1 b}$ (Figure 4(b), solid line). This trans-form is characterised by an absorption band around $240 \mathrm{~nm}$ attributed to $\pi \pi^{*}$ transitions localised on the phenyl groups, a relatively intense band around $360 \mathrm{~nm}$ due to symmetry-allowed $\pi \pi^{*}$ transitions, which are delocalised through the molecule including the two nitrogen atoms, and a weak band around $450 \mathrm{~nm}$ originating from symmetryforbidden $n \pi^{*}$ transitions occurring at the central nitrogen atoms (28). On irradiation with UV light $(365 \mathrm{~nm})$ the azobenzene unit was successfully isomerised to the $c i s$-form, where the symmetry-allowed $\pi \pi^{*}$ transitions band gets weaker and undergoes a hypsochromic shift. The band ascribed to the forbidden $n \pi^{*}$ transition also increases slightly (Figure 4(a),(b), dotted line). The photostationary state consists almost exclusively of the cis-form, as judged from changes in the $350400 \mathrm{~nm}$ spectral region, which was verified by NMR (vide infra).

On irradiating the cis-form with visible light $(450 \mathrm{~nm})$, the photochemical reversion primarily to the trans-form is effected; however, the photostationary state that is attained after a few minutes of irradiation is not exclusively trans (see Figure 4(a),(b), dashed line). For $\mathbf{1 a}$ and $\mathbf{1 b}$, the photostationary state is estimated to consist of $80 \%$ and $74 \%$ of the trans-form, respectively. The azobenzene can recover completely its original form by thermal isomerisation in the dark, showing that no photodegradation had occurred.

The reversibility of the photoreactions was tested through several irradiation cycles. The insets in
Figure 4(a),(b) show the sequential trans-to-cis and cis-totrans photoisomerisation during six cycles. Each time the same photostationary state is obtained, with only a slight difference between $\mathbf{1 a}$ and $\mathbf{1 b}$. Both $\mathbf{1 a}$ and $\mathbf{1 b}$ are thus photoswitchable and fully reversible over several cycles.

Trans-to-cis photoisomerisations of $\mathbf{1 a}-\boldsymbol{E}$ and $\mathbf{1 b}-\boldsymbol{E}$ (Figure 5(a),(c)) are almost quantitative according to recorded ${ }^{1} \mathrm{H}$ NMR spectra, see spectra of photoproducts $\mathbf{1 a}-\boldsymbol{Z}$ and $\mathbf{1 b}-\boldsymbol{Z}$ (Figure 5(b),(d), respectively), where characteristic signals of trans-forms almost fully disappear. On integrating peak areas, a yield of $94 \%$ for 1a and $\mathbf{1 b}$ is calculated, which is somewhat higher than related previously reported azobenzenophanes (72\%) (11). Quantum yields of trans-to-cis photoisomerisations of $\mathbf{1 a}$ (0.66) and $\mathbf{1 b}(0.65)$ are three times higher than that of azobenzene (circa 0.2) on irradiating the analogous absorption band (29). This difference may arise from reduced strain on the tether between extremities of the azobenzene on passing to the cis-form from the trans-form (30). Indeed, the mechanism of azobenzene photoisomerisation, which is still a source of contention, may be anticipated to receive contributions from non-sterically demanding in-plane inversion of the angle of the azonitrogen atoms or an out-of-plane rotation about the $\mathrm{N} \quad \mathrm{N}$ double bond (rotation mechanism) (8, 19, 31 35). Additionally, photoswitching does not affect the chemical shift/chemical environment of the methoxy group.

Thermal cis- to trans-isomerisations of $\mathbf{1 a}$ and $\mathbf{1 b}$ were followed spectrophotometrically at 303,313 and $323 \mathrm{~K}$. The curves tracing the kinetics of changing absorption were fit using Equation (1) (16)

$$
A \quad\left(A_{0} \quad A_{\infty}\right) \mathrm{e}^{k T}+A_{\infty}
$$

Here, $A$ is the measured absorbance, $A_{0}$ is the initial absorbance, $A_{\infty}$ is the absorbance when the thermal isomerisation (100\% of trans-form) and $k$ is the rate constant at temperature $T$. Activation energies $\left(E_{a}\right)$ were then determined from the Arrhenius equation. Thus, similar $E_{a}$ values of 99 and $101 \mathrm{~kJ} \mathrm{~mol}^{-1}$ for the thermal cis-to-trans isomerisation of $\mathbf{1 a}$ and $\mathbf{1 b}$ were noted, respectively. These values are slightly higher than that measured for parent azobenzene measured under identical conditions ( $90 \mathrm{~kJ} . \mathrm{mol}^{1}$ ), pointing to an influence of the macrocyclic tether which may disfavour the rotation mechanism of isomerisation (vide supra).

\subsection{Complexation}

As the single-crystal X-ray structure showed (Figure 3(a)), $\mathbf{1 - E}$ is less geometrically suitable than $\mathbf{1 - Z}$ to form ion inclusion complexes. ${ }^{1} \mathrm{H}$ NMR experiments (see Figure 6) show the formation of metal ligand complexes, in this case with sodium, and offer a means to measure the difference of binding strength between the trans- and cis-forms. Indeed, the singlet resonance at $3.74 \mathrm{ppm}$, attributed to the 

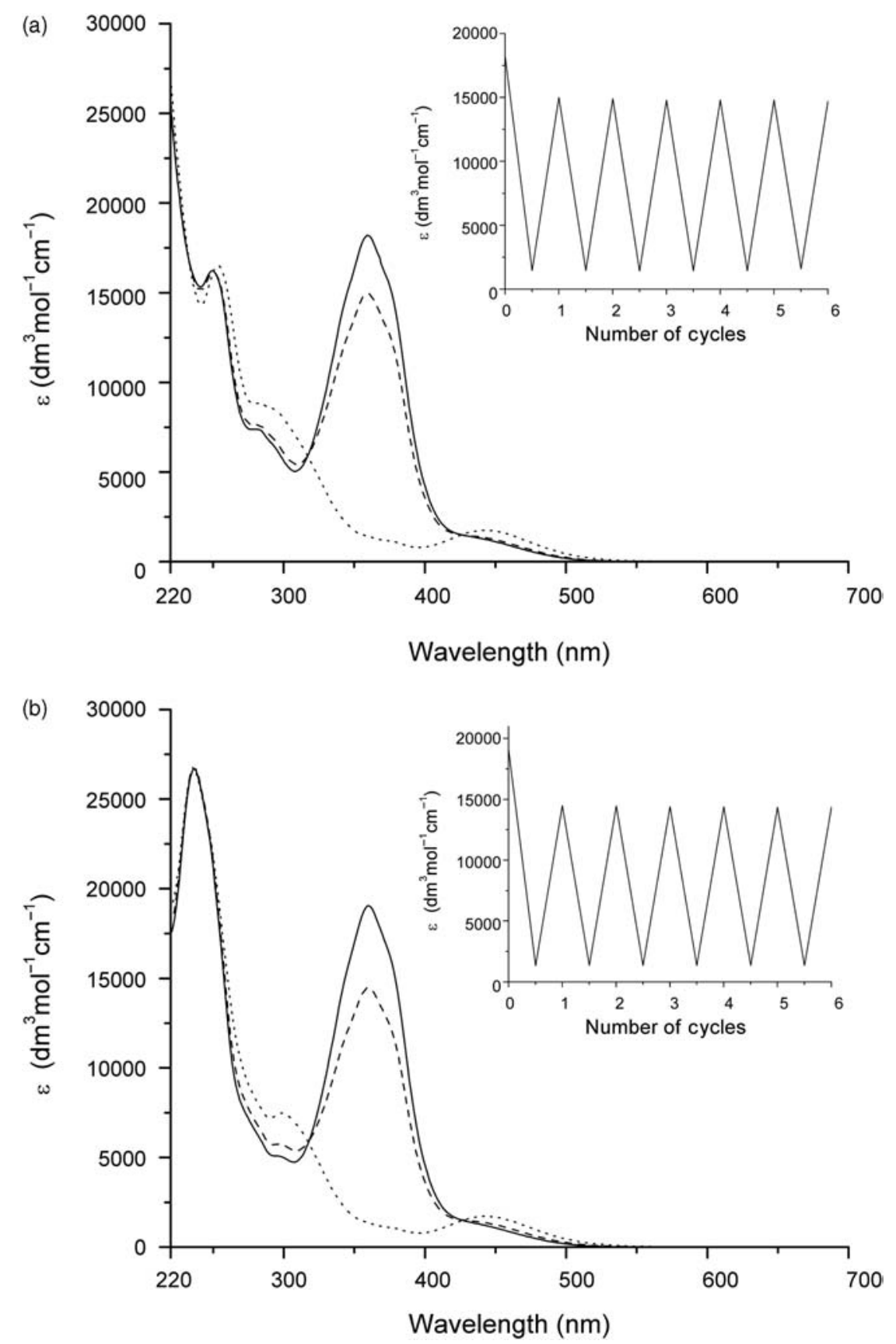

Figure 4. (a) Electronic absorption spectra of $\mathbf{1 a}-\boldsymbol{E}$ (solid line), $\mathbf{1 a}-\boldsymbol{Z}$ (dotted line) and the photostationary state after photoisomerisation $Z$ to $E$ (dashed line), (b) electronic absorption spectra of $\mathbf{1 b}-\boldsymbol{E}$ (solid line), $\mathbf{1 b}-\boldsymbol{Z}$ (dotted line) and the photostationary state after photoisomerisation $Z$ to $E$ (dashed lines). Insets: Isomerisation cycles: absorption at $365 \mathrm{~nm}$ on irradiating alternatively with UV and visible light.

methoxy group protons, is shifted further by $\mathrm{Na}^{+}$in the cis-conformation $\left(\begin{array}{llll}\Delta \delta & 0.47 \mathrm{ppm} \text { cf. } \Delta \delta & 0.18 \mathrm{ppm}\end{array}\right)$. Equally, some differences in chemical shifts for $\mathrm{CH}_{2}$ and aromatic proton resonances can be noted.

Titrations of photolariats with a range of Groups I and II metal cations (as triflate salts), and selected transition metals were performed using ${ }^{1} \mathrm{H}$ NMR spectroscopy or spectrophotometry in $\mathrm{CD}_{3} \mathrm{CN}$ or $\mathrm{CH}_{3} \mathrm{CN}$, respectively.
Indeed, the presence of potential axial binders $\mathrm{O}(\mathbf{1 a})$ or $\mathrm{S}$ (1b) is anticipated to affect the stability constants and selectivities of $\mathbf{1 a}$ and $\mathbf{1 b}$ towards different ions. In ${ }^{1} \mathrm{H}$ NMR titrations, several samples with constant concentration of photolariat and variable concentration of cation were analysed. For each signal, the variation in the chemical shift was plotted versus the concentration of cations, for example, Figure 6(e) shows the titration of 1a- 


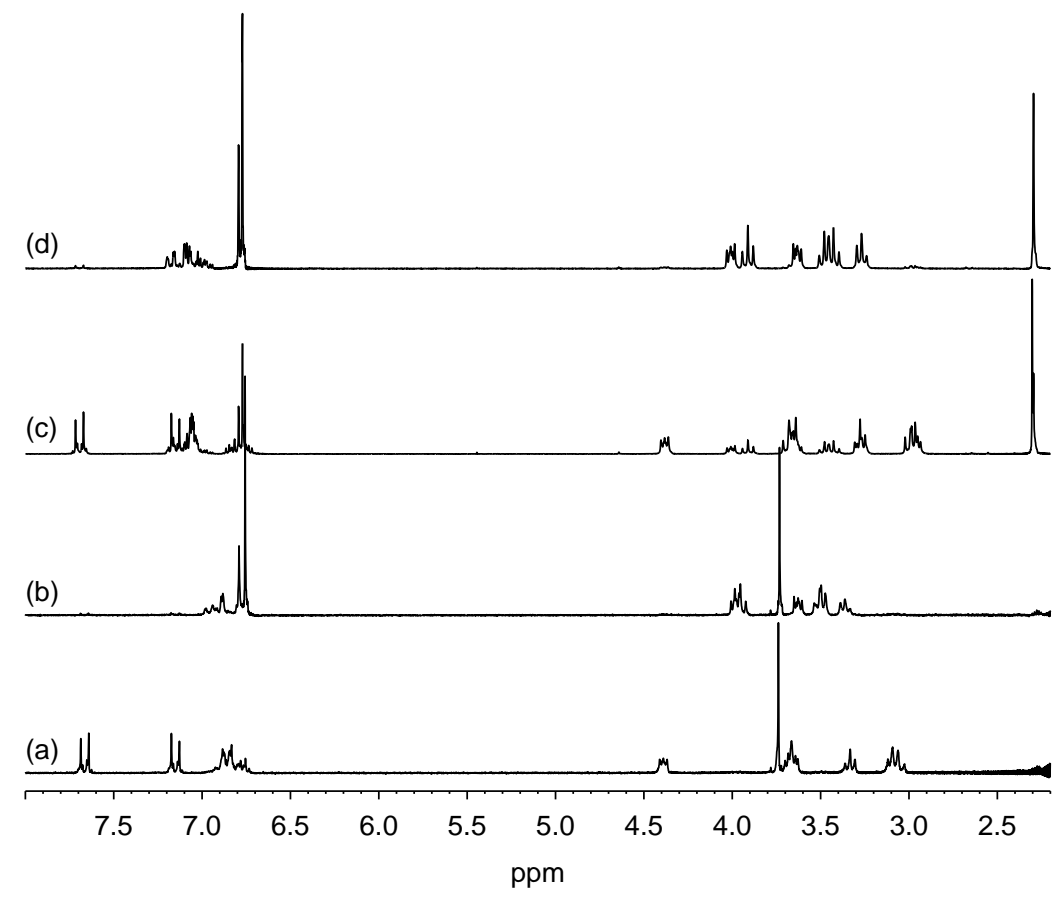

Figure 5. ${ }^{1} \mathrm{H}$ NMR spectra of photolariats (2 mM). (a) $\mathbf{1 a}-\boldsymbol{E}$, (b) $\mathbf{1 a}-\boldsymbol{Z}$, (c) $\mathbf{1 b}-\boldsymbol{E}$, (d) $\mathbf{1 b}-\boldsymbol{Z}$. $\mathbf{1 a}-\boldsymbol{E}$ and $\mathbf{1 b}-\boldsymbol{E}$ spectra were recorded before any irradiation on samples stored in the dark. $1 \mathbf{a}-\boldsymbol{Z}$ and $\mathbf{1 b}-\boldsymbol{Z}$ spectra were recorded after irradiation at $365 \mathrm{~nm}$.

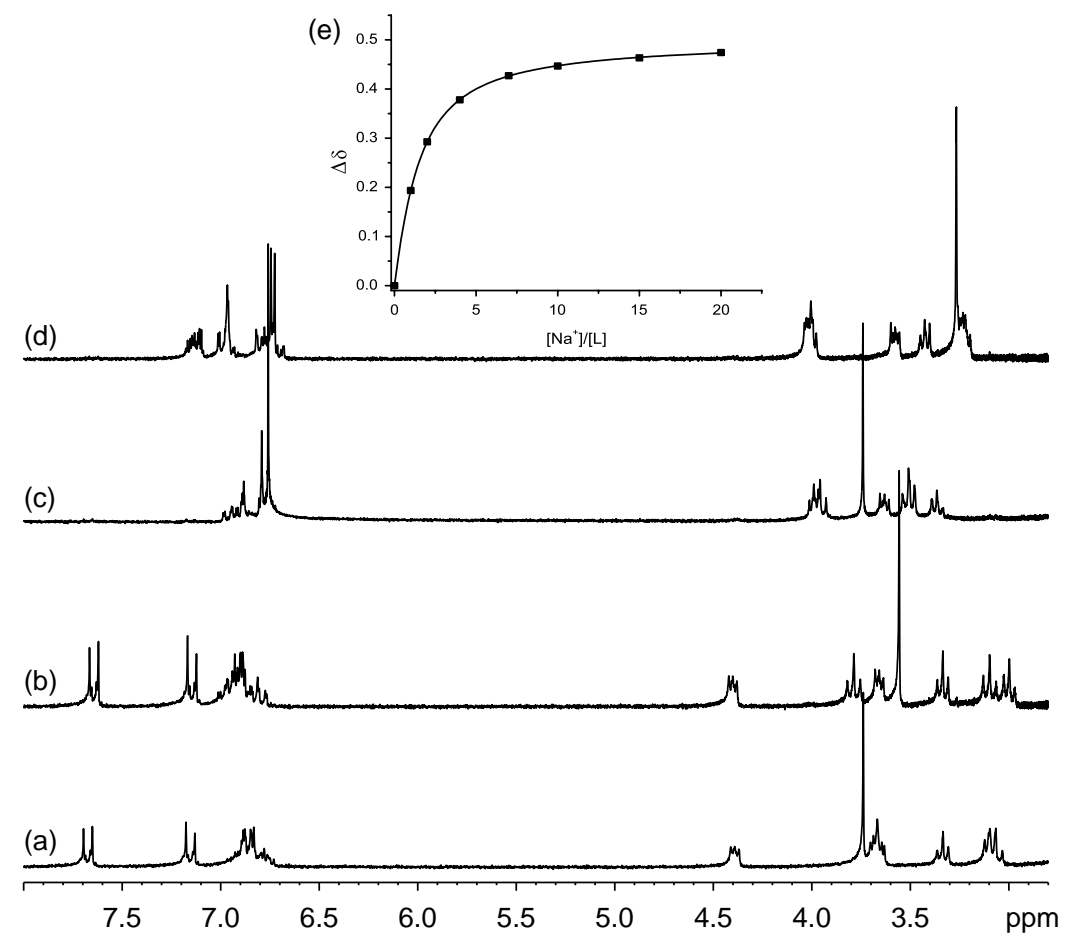

Figure 6. Complexation induced changes on ${ }^{1} \mathrm{H}$ NMR spectra of photolariat $\mathbf{1 a}$ in $\mathrm{MeCN}$ (2 mM). (a) $\mathbf{1 a}-\boldsymbol{E}$ only, (b) $\mathbf{1 a}-\boldsymbol{E}$ with 20 equivalents of sodium triflate, (c) $\mathbf{1 a}-\boldsymbol{Z}$ only, (d) $\mathbf{1 a}-\boldsymbol{Z}$ with 20 equivalents of sodium triflate, (e) ${ }^{1} \mathrm{H}$ NMR chemical shift variation in the $\mathrm{CH}_{3} \mathrm{O}$ group protons versus number of sodium per ligand. Experimental variations in the chemical shift $(\nu)$, fitted curve with calculated values ( ). 
Table 1. Photoisomerisation quantum yields, activation energies and binding constants of photolariats $\mathbf{1 a}$ and $\mathbf{1 b}$.

\begin{tabular}{|c|c|c|c|c|c|c|c|c|c|}
\hline & \multirow[b]{2}{*}{${ }^{a} \Phi_{\lambda}$} & \multirow[b]{2}{*}{${ }^{b} E_{\mathrm{a}}\left(\mathrm{kJ} . \mathrm{mol}{ }^{1}\right)$} & \multicolumn{7}{|c|}{${ }^{c} \log K_{\mathrm{a}}$} \\
\hline & & & $\mathrm{Na}^{+}$ & $\mathrm{K}^{+}$ & $\mathrm{Ca}^{2+}$ & $\mathrm{Cu}^{2+}$ & $\mathrm{Zn}^{2+}$ & $\mathrm{Tb}^{3+}$ & $\mathrm{Ag}^{+}$ \\
\hline 1a- $E$ & 0.66 & & $1.28^{\mathrm{d}}$ & $1.28^{\mathrm{d}}$ & $0^{\mathrm{d}}$ & $4.36^{\mathrm{e}}$ & $4.46^{\mathrm{e}}$ & $5.24^{\mathrm{e}}$ & \\
\hline $1 \mathrm{a}-Z$ & & 99 & $2.69^{d}$ & $2.03^{\mathrm{d}}$ & $1.7^{\mathrm{d}}$ & $4.41^{\mathrm{e}}$ & $4.67^{\mathrm{e}}$ & $5.30^{\mathrm{e}}$ & \\
\hline $1 \mathrm{~b}-E$ & 0.65 & & & & & $4.78^{\mathrm{e}}$ & $4.81^{\mathrm{e}}$ & & $4.62^{\mathrm{e}}$ \\
\hline $1 b-Z$ & & 101 & & & & $4.89^{\mathrm{e}}$ & $4.58^{\mathrm{e}}$ & & $3.94^{\mathrm{e}}$ \\
\hline
\end{tabular}

Notes: (a) Quantum yields of trans-to-cis photoisomerisation $\left(\Phi_{\lambda}\right.$ at $365 \mathrm{~nm}$ ); (b) kinetic constants of thermal cis-to-trans isomerisation; (c) logarithm of binding constants of $1 \mathrm{a}$ and 1b with different ions; (d) binding constants determined from ${ }^{1} \mathrm{H}$ NMR titrations; (e) binding constants established from spectrophotometric titrations.

$\boldsymbol{Z}$ with sodium (36). Following the spectrophotometric titrations, binding constants were extracted from a Hill plot. All binding constants are shown in Table 1 .

These studies corroborate that cis-conformations are indeed better adapted for metal ligand complex formation, and in general complexes with Groups I and II metal ions are weaker than transition metal complexes. However, a larger differentiation of binding constants between transand cis-forms is noted with the former cases, which is conducive with a higher proportion of ejected ion after isomerisation. Following this rationale, the best candidates for photoejection are sodium and calcium with 1a. On the other hand, binding constants of larger magnitude are obtained with sodium.

Concerning differences of binding affinities between 1a and $\mathbf{1 b}$ for transition metal ions tested (Table 1), a slightly higher affinity was observed with S-containing 1b with respect to O-containing 1a. Only, a relatively small difference of binding constant is observed between cis- and trans-forms. This finding can be rationalised if only one of the axial groups ( $\mathrm{OMe}$ or $\mathrm{SMe}$ ) and not all of the macrocyclic heteroatoms interact with the bound ion at any given time; thus, the small number of relatively strong interactions is surprisingly similar in the cis-and trans-forms.

\section{Conclusion}

Incorporation of additional chelating groups in the coordination sphere of azobenzene-containing crown ethers via multi-step syntheses gives photolariats, with photocontrolled binding sites. While the binding constants obtained for a range of different cations finally did not prove superior to previously reported photocrowns, the photochemistry is somewhat different, notably in terms of improved quantum yield, and a photostationary showing quasi-quantitative trans-to-cis conversion. Modifying the macrocyclic linker and axial binders is anticipated to give improved future generations of photolariats. Ultrafast studies of photoinduced isomerisations in photolariats and complexes will be reported in due course.

\section{Acknowledgements}

Financial support from the European Research Council under the European Community's Seventh Framework Programme (FP7/2008 2013) ERC grant agreement no. 208702; Région
Aquitaine; University of Bordeaux I and Ministère de la Recherche et de l'Enseignement Supérieur (A.D.; S.D.) is gratefully acknowledged.

\section{References}

(1) Lehn, J. M. Supramolecular Chemistry: Concepts and Perspectives; Wiley VCH: Weinheim, Germany, 1995.

(2) (a) Izatt, R.M.; Pawlak, K.; Bradshaw, J.S.; Bruening, R.L. Chem. Rev. 1995, 95, 2529. (b) Izatt, R.M.; Pawlak, K.; Bradshaw, J.S.; Bruening, R.L. Chem. Rev. 1991, 91, 1721. (c) An, H.; Bradshaw, J.S.; Izatt, R.M. Chem. Rev. 1992, 92, 543. (d) Izatt, R.M.; Bradshaw, J.S.; Pawlak, K.; Bruening, R.L.; Tarbet, B.J. Chem. Rev. 1992, 92, 1261. (e) Chen, X.; Izatt, R.M.; Oscarson, J.L. Chem. Rev. 1994, 94, 467. (f) Izatt, R.M.; Bradshaw, J.S.; Nielsen, S.A.; Lamb, J.D.; Christensen, J.J.; Sen, D. Chem. Rev. 1985, 85, 271. (g) Izatt, R.M. Chem. Rev. 1974, 74, 351. (h) Martell, A.E.; Motekaitis, R.J.; Motekaitis, R.J. Determination and Use of Stability Constants, 2nd ed., Wiley VCH, New York, USA.

(3) (a) Bofinger, R.; Ducrot, V.; Jonusauskaite, L.; McClenaghan, N.D.; Pozzo, J. L.; Sevez, G.; Vives, G. Aust. J. Chem. 2011, 64, 1301. (b) Baroncini, M.; Semeraro, M.; Credi, A. Isr. J. Chem. 2011, 51, 23.

(4) (a) Kao, J.P.Y.; Harootunian, A.T.; Tsien, R.Y.J. Biol. Chem. 1989, 264, 8179. (b) Li, W.H.; Llopis, J.; Whitney, M.; Zlokarnik, G.; Tsien, R.Y. Nature 1998, 392, 936.

(5) Ballardini, R.; Balzani, V.; Credi, A.; Gandolfi, M.T.; Venturi, M. Acc. Chem. Res. 2001, 34, 445.

(6) (a) Furuta, T.; Wang, S.S.H.; Dantzker, J.L.; Dore, T.M.; Bybee, W.J.; Callaway, E.M.; Denk, W.; Tsien, R.Y. Proc. Nat. Ac. Sci. 1999, 96, 1193. (b) Boyd, R.W. Non Linear Optics, 3rd ed.; Academic Press, San Diego, CA, 2008. (c) He, G.S.; Tan, L. S.; Zheng, Q.; Prasad, P.N. Chem. Rev. 2008, 108, 1245.

(7) Feringa, B.L.; Browne, W.R., Eds.; Molecular Switches, 2nd ed.; Wiley VCH: Weinheim, Germany, 2011.

(8) Dürr, H.; Bouas Laurent, H. Photochromism: Molecules and Systems; Elsevier Science: Amsterdam, 2003.

(9) Durola, F.; Dube, H.; Ajali, D.; Rebek, Jr, J. Supramol. Chem. 2011, 23, 37.

(10) Heinz, T.; Rudkevich, D.; Rebek, Jr, J. Nature 1998, 394, 764.

(11) Shinkai, S.; Minami, T.; Kusano, Y.; Manabe, O. J. Am. Chem. Soc. 1983, 105, 1851.

(12) Shinkai, S.; Ogawa, T.; Nakajima, T.; Kusano, Y.; Manabe, O. Tetrahedron Lett. 1979, 20, 4569.

(13) Shinkai, S.; Nakaji, T.; Nishida, Y.; Ogawa, T.; Manabe, O. J. Am. Chem. Soc. 1980, 102, 5860.

(14) Shinkai, S.; Manabe, O. Top. Curr. Chem. 1984, 121, 67.

(15) Shinkai, S.; Ogawa, T.; Kusano, Y.; Manabe, O.; Kikukawa, K.; Goto, T.; Matsuda, T. J. Am. Chem. Soc. 1982, 104, 1960. 
(16) Janus, K.; Sworakowski, J. J. Phys. Chem. B 2005, 109, 93.

(17) Janus, K.; Koshets, I.A.; Sworakowski, J.; Nespurek, S. J. Mater. Chem. 2002, 12, 1657.

(18) Siewertsen, R.; Neumann, H.; Buchheim Stehn, B.; Herges, R.; Näther, C.; Renth, F.; Temps, F. J. Am. Chem. Soc. 2009, 131, 15594.

(19) Lu, Y. C.; Diau, E.W. G.; Rau, H. J. Phys. Chem. A 2005 , 109, 2090.

(20) Chu, D.; Ye, T.; Wang, B. PCT Int. Appl. WO 2010065174 2010.

(21) Gansow, O.A.; Kausar, A.R.; Triplett, K.B. J. Heterocycl. Chem. 1981, 18, 297.

(22) Montalti, M.; Credi, A.; Prodi, L.; Gandolfi, M.T. Handbook of Photochemistry, CRC Press: Boca Raton, 2006.

(23) Guo, D. S.; Liu, Z. P.; Ma, J. P.; Huang, R. Q. Tetrahedron Lett. 2007, 48, 1221.

(24) Nystrom, R.F.; Brown, W.G. J. Am. Chem. Soc. 1948, 70, 3738.

(25) Zhua, Q.; Donga, L.; Niua, Y.; Xionga, C.; Liua, Y.; Liua, J.; Liua, L.; Shua, B. Polym. Bull. 2008, 61, 569.
(26) Couturier, M.; Tucker, J.L.; Andresen, B.M.; Dubé, P.; Negri, J.T. Org. Lett. 2001, 3, 465.

(27) Kinoshita, K. Bull. Chem. Soc. Jpn 1959, 32, 777.

(28) (a) Hamon, F.; Djedaini Pilard, F.; Barbot, F.; Len, C. Tetrahedron 2009, 65, 10105. (b) Dhammika Bandarab, H.M.; Burdette, S.C. Chem. Soc. Rev. 2012, 41, 1809.

(29) Bortulus, P.; Monti, S. J. Phys. Chem. 1979, 83, 648.

(30) Tahara, R.; Morozumi, T.; Nakamura, H. J. Phys. Chem. B 1997, 101, 7736.

(31) Tamai, N.; Miyasaka, H. Chem. Rev. 2000, 100, 1875.

(32) Fujino, T.; Arzhantsev, S.Y.; Tahara, T. Bull. Chem. Soc. Jpn 2002, 75, 1031.

(33) Schultz, T.; Quenneville, J.; Levine, B.; Toniolo, A.; Martinez, T.J.; Lochbrunner, S.; Schmitt, M.; Shaffer, J.P.; Zgierski, M.Z.; Stolow, A. J. Am. Chem. Soc. 2003, 125, 8098.

(34) Ciminelli, C.; Granucci, G.; Persico, M. Chem. Eur. J. 2004, 10, 2327.

(35) Diau, E.W. G. J. Phys. Chem. A 2004, 108, 950.

(36) Masiker, M.C.; Mayne, C.L.; Eyring, E.M. Magn. Reson. Chem. 2006, 44, 220. 